\title{
Thin Film Polycrystalline Silicon Nanowire Biosensors
}

\author{
Mohammad M. A. Hakim, ${ }^{*}, \dagger, \perp$ Marta Lombardini, ${ }^{*}, \dagger, \uparrow, \perp$ Kai Sun, ${ }^{\dagger}$ Francesco Giustiniano, ${ }^{\S}$ \\ Peter L. Roach, ${ }^{\S}$ Donna E. Davies, ${ }^{\|}$Peter H. Howarth," Maurits R. R. de Planque, ${ }^{\dagger, \dagger}$ Hywel Morgan, ${ }^{\dagger, \ddagger}$ \\ and Peter Ashburn ${ }^{\dagger}$ \\ ${ }^{\dagger}$ School of Electronics \& Computer Science, University of Southampton, Southampton, SO17 1BJ, U.K. \\ ${ }^{\ddagger}$ Institute for Life Sciences, University of Southampton, Southampton, SO17 1BJ, U.K. \\ ${ }^{\S}$ School of Chemistry, University of Southampton, Southampton, SO17 1BJ, U.K. \\ "Clinical and Experimental Sciences, Faculty of Medicine, University of Southampton, Southampton General Hospital, Southampton, \\ SO16 6YD, U.K.
}

Supporting Information

ABSTRACT: Polysilicon nanowire biosensors have been fabricated using a top-down process and were used to determine the binding constant of two inflammatory biomarkers. A very low cost nanofabrication process was developed, based on simple and mature photolithography, thin film technology, and plasma etching, enabling an easy route to mass manufacture. Antibody-functionalized nanowire sensors were used to detect the proteins interleukin-8 (IL-8) and tumor necrosis factor-alpha (TNF- $\alpha$ ) over a wide range of concentrations, demonstrating excellent sensitivity and selectivity, exemplified by a detection sensitivity of $10 \mathrm{fM}$ in the presence of a 100000 -fold excess of a nontarget protein. Nanowire titration curves gave antibody-antigen dissociation constants in good agreement with low-salt enzyme-linked immunosorbent assays (ELISAs). This fabrication process produces high-quality nanowires that are suitable for low-cost mass production, providing a realistic route to the realization of disposable nanoelectronic point-of-care $(\mathrm{PoC})$ devices.

KEYWORDS: Nanowires, silicon, cytokines, binding constant, immunoassay, point of care

$\mathrm{O}$ ver the past decade, silicon nanowires have emerged as important candidates for biochemical sensors. ${ }^{1-6}$ There are many reasons why silicon nanowires are of interest, including high surface-to-volume ratio, high sensitivity, and real-time, label-free detection without expensive optical components. ${ }^{1}$ Although nanowire field effect transistors have been used to sense ions, proteins, DNA, and viruses, outstanding issues remain, most notably the cost and practicality of fabrication. Therefore, the development of a very low-cost and simple fabrication route suitable for mass manufacture of disposable nanowire sensors would accelerate their uptake as point-of-care (PoC) devices. Validation of nanowire biosensors against standard enzyme-linked immunosorbent assays (ELISAs), including a characterization of binding kinetics, is an additional requirement for further development of these systems. ${ }^{2,7}$

There are two major approaches for fabricating nanowire biosensors, namely, top-down and bottom-up. Bottom-up approaches usually involve metal-catalytic nanowire growth, ${ }^{8}$ which is simple and cheap and produces uniformly sized singlecrystal nanowires, followed by an integration step such as electric field or fluid-flow-assisted nanowire positioning between lithographically defined source and drain electrodes. ${ }^{9}$ However, this introduces an additional (non-CMOS) device fabrication step and does not provide the precise control over nanowire position provided by lithographically defined nanowires. 9 Top-down approaches overcome these shortcomings, and several groups have used nanopatterning techniques such as deep-UV $\mathrm{UV}^{10}$ lithography (steppers) and electron beam lithography ${ }^{11,12}$ to fabricate silicon nanowires on silicon-oninsulator (SOI) substrates. This has the advantage of CMOS compatibility, but a serious disadvantage is the high cost associated with these advanced lithography techniques and expensive SOI wafers. To achieve a minimum feature size below $90 \mathrm{~nm}$ on a wafer scale, an enormous engineering effort has been expended to evolve stepper technology from the 365 $\mathrm{nm}$ mercury lamp to the $13.4 \mathrm{~nm}$ extreme ultraviolet regime. ${ }^{13-15}$ However, each successive stepper technology is more costly due to the requirement for more expensive optics and light sources. Furthermore, the cost of mask manufacture is also escalating, forcing chip designers to amortize design and mask expenses across the largest possible volume. Wet etch of SOI wafers has also been researched as a means of creating

Received: December 1, 2011

Revised: February 22, 2012

Published: March 20, 2012 


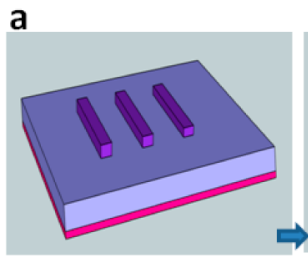

Oxide pillar formation b

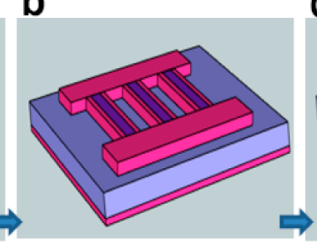

Nanowire etch

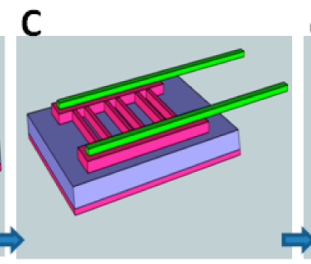

Metal contact formation

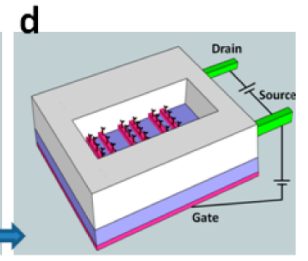

Sensing window opening

omarkers

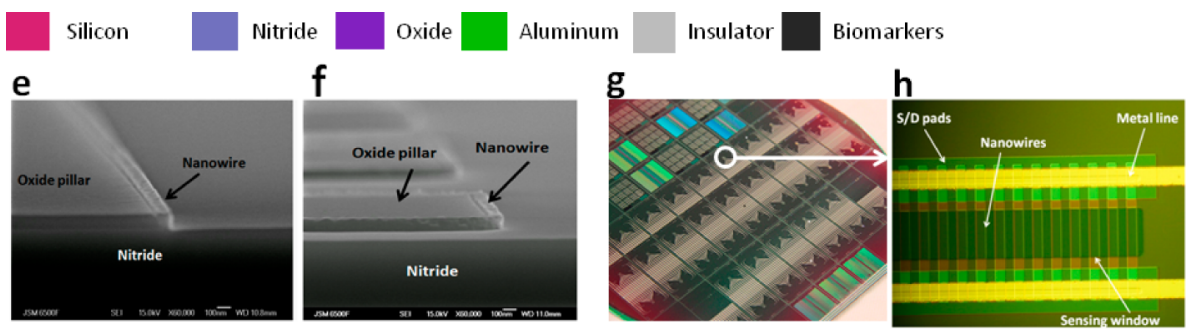

Figure 1. Schematic illustrations of polysilicon nanowire biosensor fabrication after (a) oxide pillar formation, (b) nanowire plasma etch, (c) metal contact formation, and (d) sensor window opening. The biasing configuration for the biosensor electrical measurements is also shown: (e) crosssectional SEM image of a fabricated polysilicon nanowire; (f) cross-sectional SEM micrograph of polysilicon nanowires at the corner of a pillar; (g) optical image of a completed nanowire biosensor wafer; (h) high magnification optical image of a fabricated nanowire biosensor through a sensor window.
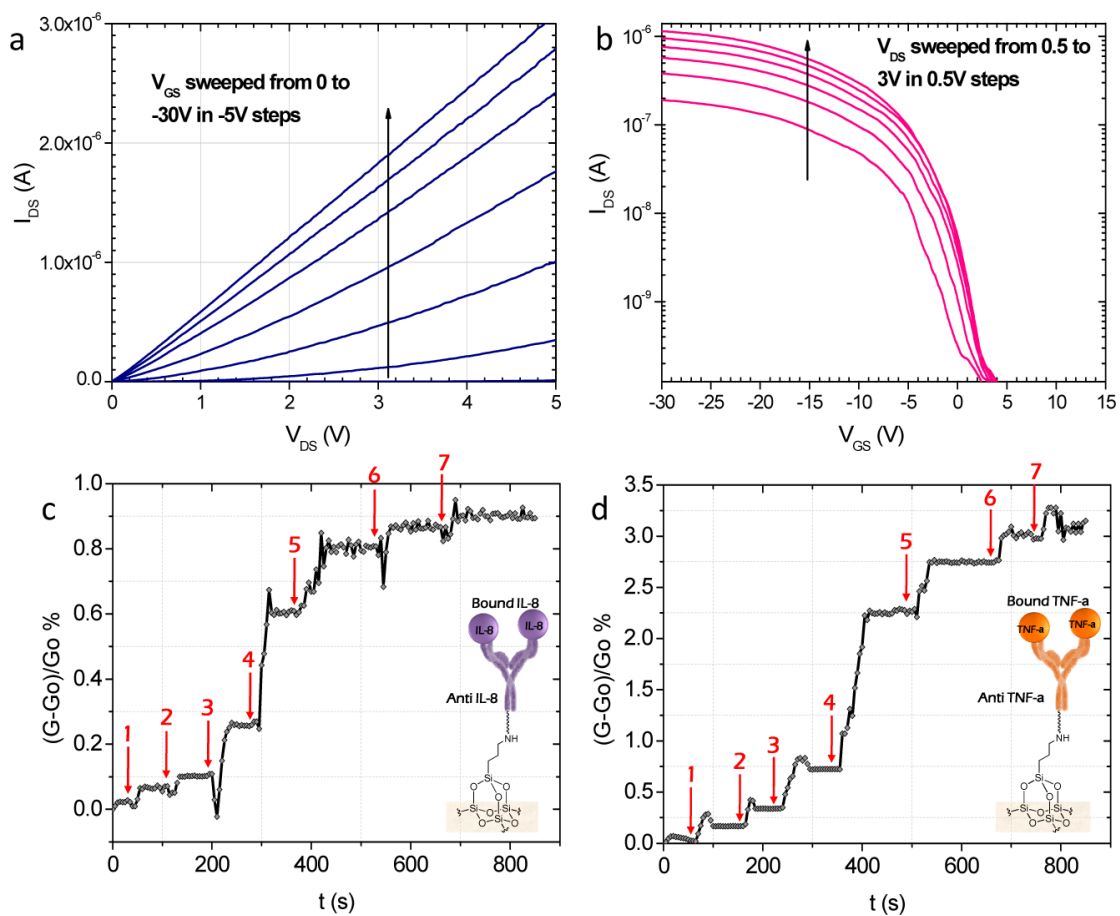

Figure 2. Polysilicon nanowire electrical characterization in air and in solution: (a) polysilicon nanowire output characteristic ( $I_{\mathrm{DS}}$ as a function of $V_{\mathrm{DS}}$ for different $V_{\mathrm{GS}}$ values) measured in air; (b) polysilicon nanowire transfer characteristic (ln $I_{\mathrm{DS}}$ as a function of $V_{\mathrm{GS}}$ for different $V_{\mathrm{DS}}$ values) measured in air; (c) normalized conduction change as a function of time showing the real-time detection of different concentrations of IL-8; (d) normalized conduction change as a function of time showing the real-time detection of different concentrations of TNF- $\alpha$. The numbers $1-7$ indicate the time at which the concentration of IL- 8 or TNF- $\alpha$ is increased by 1 order of magnitude, starting from $10 \mathrm{fM}$.

triangular nanowires without the need for advanced lithography, ${ }^{16,17}$ but as wet etching is not favored by industry, this approach does not provide a clear route to manufacturing. Recently, a low cost, top-down approach to nanowire fabrication has been reported that uses thin film technology and the spacer etch technique. ${ }^{18-20}$ This approach is particularly attractive because it produces polysilicon nanowires with nanoscale dimensions using mature lithography in combination with standard deposition and spacer etch techniques that are widely available in industry. However, the main disadvantage is that the nanowires are shaped as a quarter circle due to the absence of mask protection, which makes it difficult to control the cross-sectional area and hence the sensitivity of the nanowires.

In this paper, rectangular nanowires are fabricated using a novel spacer etch process with a thin film technology similar to that used for the manufacture of thin film transistor (TFT) displays. Using this approach, no expensive lithography tools 

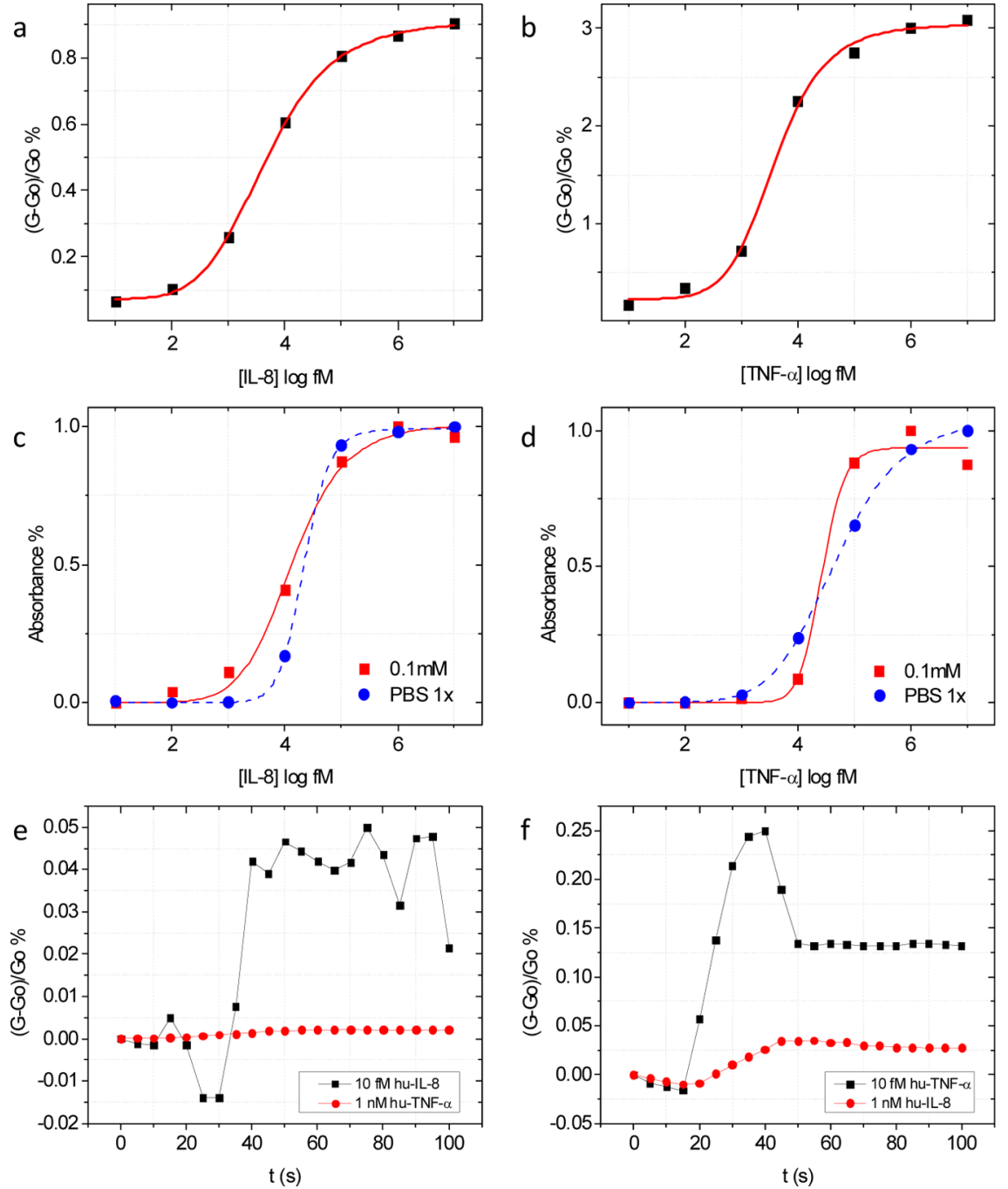

Figure 3. Polysilicon nanowire biosensor results: (a) titration curve of the reaction of IL-8 in a polysilicon nanowire biosensor (the extracted value of $K_{\mathrm{D}}$ is $4.3 \mathrm{pM}$ ); (b) titration curve of the reaction of TNF- $\alpha$ in a polysilicon nanowire biosensor (the extracted value of $K_{\mathrm{D}}$ is $4.0 \mathrm{pM}$ ); (c) detection of IL-8 using an ELISA assay at two different salt concentrations (the $K_{\mathrm{D}}$ obtained from the titration curve of the standard ELISA (square symbols) is $23 \mathrm{pM}$, while that obtained from the low salt ELISA (round symbols) gives $13 \mathrm{pM}$ ); (d) the detection of TNF- $\alpha$ using an ELISA assay at two different salt concentrations (the $K_{\mathrm{D}}$ obtained from the titration curve of the standard ELISA (square symbols) is $48 \mathrm{pM}$, while that obtained from the low-salt ELISA (round symbols) gives $26 \mathrm{pM}$ ); (e) comparison of the sensitivity of an anti-IL-8 functionalized nanowire when detecting a low concentration $(10 \mathrm{fM})$ of its specific target IL- 8 and a high concentration of a nonspecific protein $(1 \mathrm{nM}$ TNF- $\alpha$ ); (f) comparison of the sensitivity of an anti-TNF- $\alpha$ functionalized nanowire when detecting a low concentration (10 fM) of its specific target TNF- $\alpha$ and a high concentration of a nontarget protein (1 nM IL-8).

are needed and $100 \mathrm{~nm}$ nanowires can be fabricated using a simple thin film technology. Rectangular polycrystalline silicon (polysilicon) nanowires are defined by deposition and anisotropic etch using low cost reactive ion etch (RIE) equipment and mature $3 \mu \mathrm{m}$ lithography, delivering excellent control of the nanowire width and shape. Furthermore, the process can be up-scaled to produce nanowires on a very large scale similar to the production of flat screen displays. Antibodyfunctionalized nanowire sensors are fabricated and used to generate titration curves for the proteins interleukin-8 (IL-8) and tumor necrosis factor-alpha (TNF- $\alpha$ ) over a wide range of concentrations.

The nanowire biosensor fabrication starts with deposition of a $100 \mathrm{~nm}$ low pressure chemical vapor deposited (LPCVD) amorphous silicon $(\alpha$-Si) film over a silicon dioxide pillar. The oxide pillars are created by depositing a plasma enhanced chemical vapor deposited (PECVD) oxide layer, patterning using $3 \mu \mathrm{m}$ photolithography and anisotropic etching (Figure 1a). The $\alpha$-Si film was deposited at $560^{\circ} \mathrm{C}$ and doped by boron implantation at a dose of $1.5 \times 10^{13} / \mathrm{cm}^{2}$ and an energy of 25 $\mathrm{keV}$. Nanowires were formed (Figure 1b) using an Oxford Instruments Plasma Technology system $80+$ reactive ion etcher using a highly anisotropic etch, with a $\mathrm{SF}_{6}$ flow of $12 \mathrm{sccm}$, an $\mathrm{O}_{2}$ flow of $12 \mathrm{sccm}$, a pressure of $30 \mathrm{mT}$, and an RIE power of $160 \mathrm{~W}$. A $10 \mathrm{~nm}$ gate oxide was then grown at $900{ }^{\circ} \mathrm{C}$ to create a stable surface for nanowire functionalization, to crystallize the amorphous silicon to polycrystalline silicon, and to activate the implanted dopant. Aluminum contacts (Figure 1c) to either end of the polysilicon nanowire were made via heavily doped source/drain pads, and a sensing window was opened over the nanowires in a deposited insulator (Figure 1d). The silicon substrate acts as a back gate, allowing the nanowire conductivity to be modulated through the application of a back gate bias (Figure 1d). When performing biosensor measurements, the 
back gate was biased at a voltage of $-10 \mathrm{~V}$, the source was grounded, and the drain was biased at a voltage of $5 \mathrm{~V}$.

The SEM image in Figure le shows that the nanowires are rectangular in shape, with a reasonably smooth surface, few sidewall striations, and no sign of polymer particles or residues. The mean nanowire height and width are $95 \mathrm{~nm}$ and $95 \mathrm{~nm}$, respectively (20 different nanowires). Figure $S 1$ in the Supporting Information shows that the nanowire width varies very little with etch time, due to the highly anisotropic nature of the $\mathrm{SF}_{6} / \mathrm{O}_{2}$ etch. The nanowire width is therefore extremely well controlled, indicating that the technology is compatible with mass manufacture. Figure 1f shows an SEM image of the nanowire continuity at the corner of a pillar, and it can be seen that the rectangular shape is retained at the corner. Figure $1 \mathrm{~g}$ shows an image of the fabricated chips, and Figure 1h shows a high magnification image of the nanowire biosensor through the sensing window.

Figure 2a shows nanowire output characteristics in air for various values of back-gate bias. The characteristics are very linear at high values of back-gate bias (e.g., $-30 \mathrm{~V}$ ) but are less linear at low values. The $I_{\mathrm{DS}}$ value at $V_{\mathrm{DS}}=3 \mathrm{~V}$ increases from $42 \mathrm{nA}$ at $V_{\mathrm{GS}}=0 \mathrm{~V}$ to $2 \mu \mathrm{A}$ at $V_{\mathrm{GS}}=-30 \mathrm{~V}$, indicating that the back-gate bias has a significant effect on the nanowire conductance. Figure $2 \mathrm{~b}$ illustrates typical measured transfer characteristics in air for various values of drain/source bias. The characteristics are linear at low currents, with subthreshold slopes in the range of 2.3-3.0 V/decade. Polysilicon nanowires have recently been found to be very promising for nonvolatile memory design, ${ }^{21,22}$ for the design of inexpensive electrochemical sensors, ${ }^{23}$ and as sensitive nanowire-based biosensors. $^{24,25}$

The sensitivity of the nanowire biosensor is controlled by the doping concentration in the nanowire. For single-crystal silicon nanowires, all of the implanted dopant would be expected to be electrically active at room temperature and the boron dose of $1.5 \times 10^{13} \mathrm{~cm}^{-2}$ was chosen to deliver a total doping concentration of around $1 \times 10^{18} \mathrm{~cm}^{-3}$. However, for polysilicon nanowires, doping segregation at grain boundaries would be expected and hence the electrically active doping concentration is likely to be significantly less than $1 \times 10^{18}$ $\mathrm{cm}^{-3}$. To calculate the electrically active nanowire doping concentration, the hole mobility $\left(\mu_{\mathrm{h}}\right)$ was first calculated from the transconductance $\left(g_{\mathrm{m}}\right)$ using $\mu_{\mathrm{h}}=g_{\mathrm{m}} L^{2} / C V_{\mathrm{DS}}$, where $L$ is the nanowire length and $C$ is the capacitance between the nanowire and the back-gate. Using the measured $95 \mathrm{~nm} \times 95$ $\mathrm{nm}$ nanowire dimensions (Figure 1) and the $500 \mathrm{~nm}$ silicon nitride thickness gives $C=1.26 \times 10^{-16} \mathrm{~F}$ for a $10 \mu \mathrm{m}$ long nanowire and $\mu_{\mathrm{h}}=8.34 \mathrm{~cm}^{2} / \mathrm{V} \cdot \mathrm{s}$ for $V_{\mathrm{GS}}=-5 \mathrm{~V}$ and $V_{\mathrm{DS}}=0.5$ $\mathrm{V}$. The resistivity $(\rho)$ of the nanowires was found to be 34.5 $\Omega \cdot \mathrm{cm}$ at $V_{\mathrm{GS}}=-5 \mathrm{~V}$ and $V_{\mathrm{DS}}=0.5 \mathrm{~V}$, and the electrically active hole concentration $\left(n_{\mathrm{h}}\right)$ was estimated from the relation $n_{\mathrm{h}}=1$ / $\rho q \mu_{\mathrm{h}}$ for different $V_{\mathrm{GS}}$ values. The active hole concentration is found to be approximately $2 \times 10^{16} \mathrm{~cm}^{-3}$ at $V_{\mathrm{GS}}=-5 \mathrm{~V}$ and $V_{\mathrm{DS}}=0.5 \mathrm{~V}$, which is reduced to a value of $5 \times 10^{15} \mathrm{~cm}^{-3}$ at $V_{\mathrm{GS}}$ $=0 \mathrm{~V}$ and $V_{\mathrm{DS}}=0.5 \mathrm{~V}$. This value is considerably lower than the total (active plus inactive) doping concentration of around $1 \times 10^{18} \mathrm{~cm}^{-3}$ and explains why low nanowire conduction is seen in Figure $2 \mathrm{a}$ at low values of back-gate bias and why the characteristics are nonlinear. However, this low electrically active hole concentration is advantageous for nanowire biosensors, as it gives high sensitivity.

After fabrication, the nanowires were functionalized by silanizing the nanowire surface and attaching a succinic acid group that is used to covalently link one of the two different antibodies (insets in Figure $2 c, d$ ). Each step of the functionalization process was confirmed by electrically detecting a conductance change (Figure S2 in the Supporting Information). Figure $2 \mathrm{c}$ shows a stepwise titration curve for binding of the inflammatory biomarker IL- 8 to the nanowire. The normalized conductance $\left(G-G_{0}\right) / G_{0}$ is plotted as a function of time for different IL-8 concentrations, where $G_{0}$ is the conductance for a cytokine-free buffer. The IL-8 was suspended in a low-ionic-strength $(0.1 \mathrm{mM})$ phosphate buffer, and each arrow indicates an increase in IL- 8 concentration by 1 order of magnitude. An increase in normalized conductance is seen for each increase in IL-8 concentration. Whether the conductance increases or decreases upon analyte binding does not necessarily depend on the overall charge of the protein, as many charged groups are distributed through the proteins, each subjected to different screening effects from the buffer ions depending on their exact distance from the nanowire surface (see the Supporting Information). ${ }^{26}$ Figure $2 \mathrm{~d}$ shows electrical results for the sensing of the inflammatory biomarker TNF- $\alpha$. A systematic increase in normalized conductance is seen for each increase in TNF- $\alpha$ concentration. However, in this case, the magnitude of the normalized conductance increase is larger for TNF- $\alpha$ than for IL-8.

Figure 3a shows normalized conductance as a function of IL8 concentration. The black data points show the experimental data from Figure $2 c$ (see the Supporting Information). The amount of bound target $\left(\left[A_{\mathrm{b}} A_{\mathrm{g}}\right]\right)$ at steady state is a function of the concentration of free target in solution $\left(\left[A_{\mathrm{g}}\right]\right)$. The relative dissociation constant $\left(K_{\mathrm{D}}\right)$ that characterizes a specific antibody-antigen interaction was obtained by fitting the experimental data to the Hill equation: ${ }^{27}$

$$
\left[A_{\mathrm{b}} A_{\mathrm{g}}\right]=\left[A_{\mathrm{b}} A_{\mathrm{g}}\right]_{\max } \frac{1}{1+\left(\frac{K_{\mathrm{D}}}{\left[A_{\mathrm{g}}\right]}\right)^{n}}
$$

where $n$ is the Hill coefficient and $\left[A_{\mathrm{b}} A_{\mathrm{g}}\right]_{\max }$ is the maximum number of target molecules that can bind to the surface. A $K_{\mathrm{D}}$ value of $4.3 \pm 1.07 \mathrm{pM}$ is obtained for IL- 8 by fitting the curve in Figure 3a (solid line). The reliability of detection for this protein is $10 \mathrm{fM}$. The same protein was examined using a standard fluorescent ELISA experiment on microtiter plates with optical readout. The equivalent titration data for the same protein is shown in Figure $3 \mathrm{c}$, giving $K_{\mathrm{D}}=22 \pm 1.03 \mathrm{pM}$ similar to the literature value. ${ }^{28}$ This value was determined in standard nondiluted phosphate buffer, the salt concentration of which $(150 \mathrm{mM})$ is much higher than the concentration of $0.1 \mathrm{mM}$ in the nanowire buffer. The ELISA was therefore repeated using the same buffer as used for the nanowire, and the results are shown in Figure 3c, giving a slightly lower value at $K_{\mathrm{D}}=13 \pm$ $1.23 \mathrm{pM}$. Figure $3 \mathrm{~b}$ shows similar data for TNF- $\alpha$ with $K_{\mathrm{D}}=4.0$ $\pm 1.19 \mathrm{pM}$ (Figure $3 \mathrm{f}$ ), with a similar reliability of detection at $10 \mathrm{fM}$. Figure $3 \mathrm{~d}$ shows ELISA data in high and low salt concentrations, giving $K_{\mathrm{D}}=48 \pm 1.05$ and $26 \pm 1.28 \mathrm{pM}$, respectively, close to the literature value of $70 \mathrm{pM}^{29}$ The lowsalt ELISA $K_{\mathrm{D}}$ is again in reasonable agreement with that measured from the nanowires. This decrease in the value of $K_{\mathrm{D}}$ is not unexpected because the on-rate of an antibody interaction can increase as the ionic strength is reduced, since the electrostatic interactions are screened to a lesser extent. ${ }^{30,31}$ This demonstrates that the requirement to operate at low ionic 
strength is not perturbing the antibody binding constant in a significant way.

The selectivity of this nanowire assay was investigated by comparing the cross-reactivity of two nanowires functionalized with the two different antibodies against nontarget cytokines. The normalized conductance of a functionalized nanowire was measured when exposed to $1 \mathrm{nM}$ nonspecific target protein. Anti-IL-8 functionalized nanowires were exposed to $1 \mathrm{nM}$ TNF- $\alpha$ (Figure 3e) and anti-TNF- $\alpha$ functionalized nanowires to $1 \mathrm{nM}$ IL-8 (Figure $3 \mathrm{f}$ ). In both cases, the conductance change in the presence of high concentrations of the nonspecific target is at least $0.03 \%$ smaller than the equivalent response for a $10 \mathrm{fM}$ concentration of target antibody. These results indicate that the nanowire biosensor has high specificity and sensitivity to the target proteins even in the presence of a large concentration of a nonspecific target.

In conclusion, we have developed a novel and simple topdown fabrication process to manufacture high quality nanowires from polysilicon. This process is compatible with mass manufacture and produces rectangular nanowires with excellent control of nanowire width. The polysilicon nanowire biosensors have been evaluated with two different cytokines, IL- 8 and TNF- $\alpha$, giving dissociation constants that are in agreement with ELISA measurements in low ionic strength buffers. This validates the original concept of developing nanoscale field effect transistors for low-cost parallel biochemical assays including point-of-care disposable systems.

\section{ASSOCIATED CONTENT}

\section{S Supporting Information}

Additional description of the system and experimental procedure. This material is available free of charge via the Internet at http://pubs.acs.org.

\section{AUTHOR INFORMATION}

\section{Corresponding Author}

*E-mail: mmah@ecs.soton.ac.uk (M.M.A.H.); ml09v@ecs. soton.ac.uk (M.L.).

\section{Author Contributions}

${ }^{\perp}$ These authors contributed equally to the work.

\section{Notes}

The authors declare no competing financial interest.

\section{ACKNOWLEDGMENTS}

The authors acknowledge the support of the Engineering and Physical Sciences Research Council (EPSRC).

\section{REFERENCES}

(1) Chen, K.; Li, B.; Chen, Y. Nano Today 2011, 6, 131-154.

(2) Ramgir, N. S.; Yang, Y.; Zacharias, M. Small 2010, 6, 1705-1722.

(3) Lee, M.; Baik, K. Y.; Noah, M.; Kwon, Y.-K.; Lee, J.-O. Lab Chip 2009, 9, 2267-2280.

(4) Curreli, M.; Zhang, R.; Ishikawa, F. N.; Chang, H.-K.; Cote, R. J.; Zhou, C.; Thompson, M. E. IEEE Trans. Nanotechnol. 2008, 7, 651667.

(5) Zheng, Z; Patolsky, F.; Cui, Y.; Wang, W. U.; Lieber, C. M. Nat. Biotechnol. 2005, 23, 1294-1301.

(6) Stern, E.; Klemic, J. F.; Routenberg, D. A.; Wyrembak, P. M.; Turner-Evans, D. B.; Hamilton, A. D.; LaVan, D. A.; Fahmy, T. M.; Reed, M. A. Nature 2007, 445, 519-522.

(7) Arlett, J. L.; Myers, E. B.; Roukes, M. L. Nat. Nanotechnol. 2011, 6, 203-215.
(8) McAlpine, M. C.; Friedman, R. S.; Jin, S.; Lin, K. H.; Wang, W. U.; Lieber, C. M. Nano Lett. 2003, 3, 1531-1535.

(9) Lu, W.; Xie, P.; Lieber, C. M. IEEE Trans. Elecron. Dev. 2008, 55, 2859-2876.

(10) Gao, Z.; Agarwal, A.; Trigg, A. D.; Singh, N.; Fang, C.; Tung, C. H.; Fan, Y.; Buddharaju, K. D.; Kong, J. Anal. Chem. 2007, 79, 32913297.

(11) Park, I. Z.; Li, Z.; Pisano, A. P; Williams, R. S. Nanotechnology 2010, 21, 1-9.

(12) Bunivomich, Y. L.; Shin, Y. S.; Yeo, W. S.; Amori, M.; Kwong, G.; Heath, J. R. J. Am. Chem. Soc. 2006, 128, 16323-16331.

(13) Schellenberg, F. IEEE Spectrum 2003, 40, 34-39.

(14) Nowak, E. IBM J. Res. Dev. 2002, 46, 169-180.

(15) Miller, R. E.; Bischoff, P. M.; Sumner, R. C.; Bowler, S. W.; Flack, W. W.; Fong, G. Proc. SPIE 2000, 4000, 567-578.

(16) Chen, S.; Bomer, J. G.; Wiel, W. G. V.; Carlen, E. T; Berg, A. V. ACS Nano 2009, 3, 3485-3492.

(17) Lee, M. H.; Lee, K. N.; Jung, S. W.; Kim, W. H.; Shin, K. S.; Seong, W. K. Int. J. Nanomed. 2008, 3, 117-124.

(18) Lin, H.-C.; Lee, M. H.; Su, C. J.; Huang, T. Y.; Lee, C. C.; Yang, Y. S. IEEE Electron Device Lett. 2005, 26, 643-645.

(19) Hsiao, C. Y.; Lin, C. H.; Hung, C. H.; Su, C. J.; Lo, Y. R.; Lee, C. C.; Lin, H. C.; Ko, F. H.; Huang, T. Y.; Yang, Y. S. Biosens. Bioelectron. 2009, 24, 1223-129.

(20) Su, C. J.; Lin, H. C.; Tsai, H. H.; Hsu, H. H.; Wang, T. M.; Huang, T. Y.; Ni, W. X. Nanotechnology 2007, 18, 215205.

(21) Hung, M. F.; Wu, Y. C.; Tang, Z. Y. Appl. Phys. Lett. 2011, 98, 162108.

(22) Wu, Y.-C.; Hung, M.-F; Su., P.-W. J. Electrochem. Soc. 2011, 158, H578-H582.

(23) Chen, H. Y.; Lin, C. Y.; Chen, M. C.; Huang, C. C.; Chien, C. H. Jpn. J. Appl. Phys. 2011, 50, 04DL05.

(24) Lin, C.-H.; Hung, C. H.; Hsiao, C. Y.; Lin, H. C.; Ko, F. H.; Yang, Y. S. Biosens. Bioelectron. 2009, 24, 3019-3024.

(25) Lu, M. P.; Hsiao, C. Y.; Lai, W. T.; Yang, Y. S. Nanotechnology 2010, 21, 425505.

(26) Vico, L. De.; Iversen, L.; Sorensen, M. H.; Brandbyge, M.; Nygard, J.; Martinez, K. L.; Jensen, J. H. Nanoscale 2011, 3, 36353640.

(27) Jiang, X.; Weise., S.; Hafner, M.; Rocker, C.; Zhang, F.; Parak, W. J.; Nienhuas, G. U. J. R. Soc., Interface 2010, 7, S5-S13.

(28) Kurdowska, A.; Miller, E. J.; Noble, J. M.; Baughman, R. P.; Matthay, M. A.; Brelsford, W. G.; Cohen, A. B. Immunology 1996, 157, 2699-2706.

(29) Weinberg, J. M.; Buchholz, R. Medical 2006, 28-29.

(30) Squires, T. M.; Messinger, R. J.; Manalis, S. R. Nat. Biotechnol. 2008, 26, 417-426.

(31) Schreiber, G. Curr. Opin. Struct. Biol. 2002, 12, 41-47. 